\title{
Microcomputer system for multistep specimen processing and reporting in a microbiology laboratory
}

\author{
R ASHLEY, B DOWNING \\ From the Department of Microbiology, Good Hope General Hospital, Sutton Coldfield, West Midlands B75 \\ $7 R R$
}

SUMMARY A microcomputer system for use in the sequential processing of specimens has been developed in a hospital microbiology department which receives 90000 specimens a year. Data handling is performed directly through the computers without the use of cards or worksheets. The system facilitates the multistep processing of specimens and the servicing of results inquiries and provides an alphabetical daysheet of completed and incomplete work, report form printout, and automatic transfer of reported data to an existing on line database. The system includes on line break point antibiotic sensitivity testing and multipoint agar organism identification. The hardware was purchased as required at small cost per unit, and the software was written by the authors. More than $80 \%$ of specimens received are processed using the computers, the remainder being under development. The system has been running for three years without major breakdown.

Computer systems have been used for a long time in haematology and biochemistry laboratories because they allow accurate, reliable, and speedy data processing. Data processing in microbiology differs from that in other laboratories because the data are largely textual, and the results are obtained in a multistep fashion. ${ }^{1-5}$ Various data processing systems have been described. Some use punched cards with off site processing ${ }^{6-9}$ and others use either a self contained laboratory compute $r^{-6}$ or one connected on line to a hospital main frame computer. ${ }^{31011}$ These systems have been developed for large laboratories, usually handling more than 100000 specimens a year, with the emphasis being on data storage for statistical and epidemiological purposes. They may be inefficient since results entered by hand on to laboratory work cards have to be reentered into the computer (often by non-technical staff so that the final report must be carefully checked against the original). Microcomputers have recently been used for data processing in smaller laboratories ${ }^{1213}$ and for more specific tasks. ${ }^{14-16}$

The system described in this paper facilitates the sequential processing and reporting of specimens within a microbiology laboratory.

Accepted for publication 13 September 1984

\section{Objectives}

The following objectives were required of the system.

1 Each specimen processing area should be supported by a largely autonomous computer subsystem, so that the whole system is not totally reliant on any one unit of computer equipment.

2 The system should be developed and introduced in stages: the hardware purchased in small cost units as required, and the software assimilated into the laboratory with little change to existing work patterns. It should be developed by at least two members of the laboratory staff to allow full cover for system maintenance and changes in routine-for example, alterations in antibiotic reporting.

3 It should allow all staff easily to adapt to, understand, and be in control of the system without specialised training. Data entry should be uncomplicated, and the use of codes and other restrictions should be kept to a minimum. All relevant instructions must be displayed on the computer screen, with full error trapping and explanatory prompts in the data entry sections. The patient details should be entered easily by existing clerical staff or technical staff as required.

4 The system must be as flexible as possible, with results keyed into the computer(s) directly as the work is processed and allowing result amendments 
and report printout at any time of the day. The system must be capable of easily generating provisional reports, extra copies of reports, and non-standard reports.

5 Work processing should be speeded up by eliminating filing, photocopying, manually prepared daysheets, and the repeated manual transcription of results.

6 Reports, daysheets, and visual display unit (VDU) formats should be clear and concise with daysheets in surname alphabetical order including the progress of unfinished work.

7 It should be possible to revert easily to a manual system in the event of major breakdown.

\section{System description}

Good Hope Hospital is a district general hospital with 550 beds which also services outlying hospitals with another 1658 beds. The microbiology department processes 90000 specimens a year, of which $45 \%$ are from general practitioners.

The previous system at Good Hope Hospital entailed the separation of specimens into seven processing types: faeces, urines, blood cultures, serology, sputa, TB, and general bacteriology. Specimen investigations were noted on the reverse of the request form and final results were applied to the front with the use of rubber stamps. Photocopies of the reports were issued and the original forms were stored in filing cabinets for a minimum of three months. Typed daysheets were generated for each specimen type, with results manually entered as reports were completed.

The microcomputer system described here assists in the progressive processing of specimens on the urine and general bacteriology benches, which comprise $81 \%$ of all specimens received. The system

\section{GENERAL BACTERIOLOGY PROGRAMS MENU}

First entry of the day.

Patient details and micro......................................

Culture entry ....................................................

Antibiotic sens data ..............................................

Antibiotic sens reading ..........................................

Organism identification ........................................F

Report form printout ............................................

Daysheet printout .................................................

Result enquiry .....................................................

URINE BACTERIOLOGY PROGRAMS MENU

First entry of the day ............................................

Patient details, micro etc .......................................

Data backup ...........................................................

Inhibitory substance results....................................

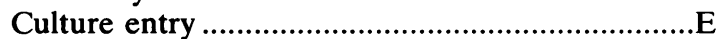

Report form printout ........................................... also includes on line break point antibiotic sensitivity testing and multipoint agar organism identification.

Software for the remaining benches is under development.

\section{Hardware}

The microcomputer system described consists of the following: four microcomputers-Commodore 8032 (32K RAM with 80 by 25 column screen); two dual drive floppy disc units-Commodore 8050 (each of 2 by $500 \mathrm{~K}$ storage); and two dot matrix printersAnadex DP9501 (120 characters per second speed).

The purchase price of the hardware was $£ 7000$ (1984 prices) spread over four years and is covered by a maintenance contract costing $£ 925$ a year.

The urine bench uses one microcomputer, disc drive, and printer. The general bacteriology, antibiotic, and identification benches require three microcomputers and share one disc drive and printer using the Multipet System (Taylor-Wilson Systems, Solihull, England).

\section{Software}

The computerised system was developed for the particular needs of the laboratory and the application programs were written in Commodore Basic 4.0 by the authors. No commercial software was purchased. There are two master programs menus, one for general bacteriology and one for urines. Each main program offers subsiduary menus. All programs are loaded from disc using a single key tap, with a return to the master menu on completion.

The average loading time for a program is $9 \mathrm{~s}$. The programs menus offer the following options.

Data backup

Transfer to database........................................

File status.

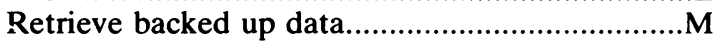

Print misc lab labels ...........................................

Microbiology accounts ..........................................

One day analysis statistics......................................

Gardnerella listing...............................................

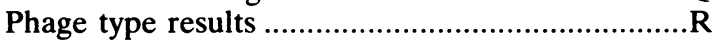

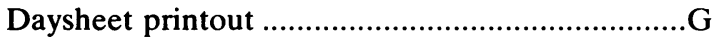

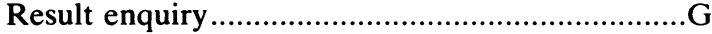

ANC dipslide culture results .................................

File status .................................................................

Retrieve backed up data .......................................

One day analysis statistics .................................... 
The programs menus also contain auxiliary programs such as microbiology accounts, which do not form part of the routine processing system described here.

Up to 250 bytes are available for each specimen for free text comments on the VDU or report form. In most instances data are recorded on to disc directly as they are entered at the keyboard, and little is held in volatile memory. The use of numerical codes and abbreviations for entry of results-for example, culture result text-is kept to a minimum, but where used they are immediately converted to full text on the screen. To safeguard against loss of data, programs were written to enable the rapid and convenient copying of files from the in use disc on to auxiliary files in the second drive.

\section{ERROR TRAPPING}

Comprehensive checks are employed in all data entry routines to avoid errors and nonsense data. Each individual key press is checked and invalid non-alphanumeric keys are ignored. The final composite entry is also checked for general legality (length etc) and by more specific criteria which vary with each input request-for example, a routine accessed by laboratory reference number will accept only a five digit number within predetermined limits. The entry of erroneous data results in an audible bleep and a VDU reminder of the requirements.

\section{FORMATTING}

Much attention was given to producing VDU, report form, and daysheet formats, which were clear and concise. Any abbreviated or coded keyboard entries immediately appear as decoded text on the VDU. Report forms and daysheets are printed in full text without codes. All data entry sections display patient details and all previously entered results including free text comments.

\section{General bacteriology specimen processing}

\section{FIRST ENTRY OF THE DAY PROGRAM}

At the start of a new working day the date and first laboratory reference number are entered using the first entry option from the general bacteriology programs menu. On receipt of a specimen batch in the laboratory, matched specimens and request forms receive an adhesive laboratory reference label bearing a specimen type prefix and a unique five digit number. Cultures and preparations for microscopy are set up for each specimen dependent on the site and clinical information supplied.

PATIENT DETAILS AND MICRO PROGRAM

This option is chosen next from the programs menu and the following subsidiary menu is displayed:

Patient details entry

Wet prep results

Gram film results

Free text for report form

Amend/repeat

Programs menu

\section{Patient details entry}

For each specimen in turn this option generates a sequential laboratory reference number and then requests the specimen type and patient information. Specimen/site is entered by tapping a two digit number from a list on the VDU. A miscellaneous specimen choice is available for unusual sites and a free text description is requested. Generalised sites such as ulcer will also require further description. Swab specimens obtained from postoperative wounds automatically request control of infection details, and cerebrospinal fluid specimens require appearance, cell count, leucocyte differential, and protein estimation to be entered.

The main patient details are then entered, and the following information requested: name, sex, age, registration number, address for report, consultant/ general practitioner, chemotherapy, and clinical details. " $R$ " may be entered at any stage to permit a repeat of the previous item. As with all other programs, illegal entries result in an audible bleep and a visual prompt of the required format. Where multiple specimens from one patient are received, patient details may be duplicated automatically.

On completion of each patient details entry, four options are displayed.

1 Comment box

2 Next specimen

3 Repeat all entries

4 Return to patient details menu

The comment box option offers the following possibilities: a copy of the report to be sent to an extra address; free text comments on the VDU for all subsequent displays; free text comments for the report form; report form to receive "specimen inadequately labelled" comments; private patient copy of report and file marker to be generated. After checking the entered details, the next specimen request may be called. A repeat of all patient details for the current specimen may be made. A return to the patient details menu may be made at any time. After entry of the patient details, the request form is no longer required, and all subsequent processing is performed directly through the microcomputer(s).

Wet preparations

This section allows microscopy results for 
trichomonas and yeasts to be entered on appropriate specimens. The occasional atypical specimen requiring a wet preparation may still have the results entered using a routine accessed by the laboratory reference number.

\section{Gram stain films}

Results are requested only on appropriate specimens and are entered for each organism type quantitatively using $0,1,2$, or 3 . Specimens for Gram negative diplococci screen automatically require a response to "GC seen ? - yes/no"; a positive entry requires verification before the program continues. Free text comments on the Gram film may be made for VDU display on all subsequent processing.

Free text for printing on the report form may also be entered directly from the patient details menu, allowing the reporting of unusual requests-for example, crystals in fluids

\section{Amend/repeat}

This section allows an individual record or block of records to have patient details or microscopy results, or both, altered or re-entered completely.

\section{Programs menu}

A return to the programs menu may be made at any time during the entry of a batch. A subsequent recall of any section within this program will allow direct continuation from the last entry.

\section{CULTURE ENTRY PROGRAM}

This program choice offers the following menu:

1 Enter first day culture results

2 Enter unfinished work culture results

3 Amend a culture result

4 Programs menu

1 As the culture plates from each specimen are read, the patient details, microscopy results, and any internal VDU comments are displayed on the screen. Culture results may be entered in two ways: (a) as text codes-for example, 01 = no growth, 07 $=$ Gardnerella not isolated; $(b)$ as growth amount, entered as $1,2,3$, or a viable count. Text codes are translated immediately to full text format on the screen. After entry of a growth amount, the organism identity is requested. This can be either a logical four letter mnemonic-for example, PRMI = Proteus mirabilis - or the organism name in full. If the identity is not yet known a ? is entered. Any further tests are then requested. For the common isolates which require a fixed set of investigations a standard abbreviation may be entered resulting in full text conversion. Free text comments for the report form and provisional reports containing free text may also be generated at this stage. Cultures which require further investigations generate a further work form printed out on the sensitivity bench, which contains all the patient details and previous results together with the further work to be done. These forms are normally used only for initial reference in inoculating broths for sensitivities etc.

2 This option allows for unfinished work entry from previous days. All the usual details are displayed on the screen together with any previously entered culture results, sensitivities, free text report form comments, and details of any provisional report which may have been issued. Previous culture result entries may be completely overwritten or results may be partially amended. Organism identity, sensitivities, culture text, and further work may all be amended individually.

3 Culture results are normally read into the computer in batches. This menu choice allows a single previously incomplete record to be accessed directly for amending.

4 A return to the culture entry menu may be made at any time.

Most sensitivity results are automatically recorded on to the patient files from the break point sensitivity program, but results may be entered manually in this section if required-for example, anaerobes.

\section{ANTIBIOTIC SENSITIVITY TEST READING PROGRAM}

Antibiotic sensitivity testing on all aerobic nonurine isolates is performed by a break point technique using Adatabs (Mast Laboratories, Bootle, Merseyside). For simplicity, a single comprehensive batch of plates is used for all isolates and the report form program selects only relevant antibiotics.

Suitably diluted 4-6 h broth cultures are applied to the set of break point plates using a 31 pin multipoint inoculator. DNase and $\beta$-lactamase test plates are included.

Plates are read in the morning at the same time as primary cultures and organism identities are read on other microcomputers. The program starts by loading the required isolate data, which are recorded automatically on the previous afternoon using the "antibiotic sens data" program. Access to the disc drive is not then required until the general bacteriology bench is ready to accept the sensitivity results. For each antibiotic plate in turn, an image of the plate with reference data is shown on the VDU. As each growth or no growth reading is entered with a single key stroke, the VDU image receives a dot or asterisk in reflection. The image allows a rapid and accurate check before the next plate is called.

After each antibiotic plate has been read, readings are interpreted and attention may be drawn to any unusual or atypical results. The plates for detection 
of DNase and $\beta$-lactamase production are read in a similar manner. DNase test results are used by the computer to identify coagulase negative staphylococci, and $\beta$-lactamase test results are used to verify the sensitivity of staphylococci and haemophili to penicillins.

Organism identification is then requested for coliforms and streptococci. A tabular paper printout is next obtained of all sensitivity results together with a list of any unusual or atypical results. Results may be amended or deleted at this stage, before automatic transfer to the appropriate patient files on disc.

\section{ORGANISM IDENTIFICATION PROGRAM}

A multipoint inoculated agar plate identification scheme using Mast ID (Mast Laboratories) is used for most enterobacteriaciae. The results are read directly into the computer in a similar manner to the antibiotic sensitivities. For each isolate in turn a list of results is printed on the VDU together with the identification.

\section{REPORT FORM PRINTOUT PROGRAM}

After the culture plates have been read, provisional, final, or additional reports are generated within the laboratory on preprinted stationary. Although all sensitivity results are recorded; only relevant results are printed by the program. Report forms may be generated at any time of the day.

\section{DAYSHEET PRINTOUT PROGRAM}

Daysheets in surname alphabetical order are produced once a day after report form printout. They are formatted for ease of reference and contain the patient details, all results, the current state of work of unfinished reports, and any additional comments which may have been printed on provisional or final reports. Completed reports have the date of issue noted.

At the end of the daysheet a list of all postoperative wound swabs is produced, followed by a list of all inpatient Staphylococcus aureus and Pseudomonas aeruginosa isolates requiring typing. The details of all inpatient Staph aureus isolates are also saved on a separate file for entry of phage type results at a later date. A printout of these results is obtained automatically every Friday.

After this, the daysheet program automatically reorganises the files in readiness for the new day's work by: (a) transferring positive completed records to a separate file in readiness for transfer to the database; (b) saving unfinished records; and (c) erasing all completed and reported records.

The system is programmed each year with the dates of the once monthly work load statistics analysis. The daysheet program will automatically calculate and produce a paper printout of these figures on the required days. This printout may also be obtained at any other time if required.

\section{RESULTS INQUIRY PROGRAM}

Since the daysheet records both incomplete and final results and lists names alphabetically, inquiries may be answered quickly and easily. A results inquiry facility is also available on the computers at bench level for work still being processed. A search is made with the full name, surname only, or the first two letters of the surname, until the search is successful. A VDU and paper printout is obtained in case of multiple specimens from one patient. A search of 100 records takes only $12 \mathrm{~s}$.

\section{DATA BACK-UP PROGRAM}

The up to date records of all patient details and results are copied from the in use disc on to auxiliary files on the second drive after each batch of work has been processed. The back-up program runs automatically after the choice from the programs menu has been made. All of the data may be copied or just the latest batch of work.

\section{TRANSFER TO DATA BASE PROGRAM}

All positive records are automatically transferred for long term store once a day to an existing on line database comprising a Televideo microcomputer running Micromumps language, 7.5 megabyte disc store, and printer (purchase price £5400). The database produces all control of infection data, Koener type statistics, and antibiotic sensitivity statistics. These data are produced on hard copy and stored in condensed form on floppy disc for archiving.

Access to this database is restricted by personal security codes.

\section{Urine specimens}

Urine specimens are processed with the aid of a similar but autonomous microcomputer system, which comprises one microcomputer, dual disc drive, and printer. Patient details, microscopy, and culture results are entered and report form, alphabetical daysheet, result inquiry, and workload statistics facilities are available in a similar manner to the general bacteriology. Antibiotic sensitivity testing of urine isolates is performed by disc technique and results are entered directly into the computer at the culture entry section.

\section{Discussion}

Most microbiology computer systems are devoted to 
long term storage and analysis of data and have little or no impact on work under process. ${ }^{1-10}$ We believe that a microbiology computer system should ideally also assist in the more practical tasks of processing specimens in a busy routine laboratory.

Database systems normally use mini or mainframe computers because of their larger storage capacities and faster operating speeds. Microcomputers, however, have the advantages of flexibility and low cost. During specimen processing operating speed has not been a problem since data are not captured from on line automated machinery. Program routines in machine code would be a solution if this need arose. The potential queuing problems caused by microcomputers sharing peripheral devices have been largely avoided by appropriately designed programs. Memory size has not been a problem since the Commodore floppy disc drives allow up to one megabyte of storage for data under process and programs, and we see no limitation in handling any future increase in workload.

Since our system consists largely of autonomous sub-systems for each specimen processing area, it was developed in stages at our own pace, and the hardware was purchased in small cost units when required. None of the laboratory staff had any previous experience of computers, but they have accepted the system well. This we think is due to the gradual implementation of the system, and software written by the authors which is tailored to the specific practical requirements of the laboratory staff. The resultant increase in speed and efficiency of specimen processing has been noted by all the laboratory staff. Changes in routine or requirements of the staff are easily catered for. Much time and effort was given to developing a system which was simple to understand and operate with little training. To this end, relevant instructions are given in all sections, and a help facility is available where required. The comprehensive error trapping not only rejects erroneous data but also prompts the operator of the correct requirements.

Prompts of this kind are also installed in other sections, such as the occurrence of an unusual or invalid sensitivity pattern or the attempt to report results which contradict a previous provisional report. Since much microbiology data is textual, coded or abbreviated computer entries are often required to permit speedy processing. We have attempted to limit the use of results codes to a minimum commensurate with rapid and accurate entry, but where used they are invariably logical abbreviations which are immediately validated and translated into full text on the VDU. In many instances a single key tap may be used to enter data for example, $>$ equating to more than 100000 organisms per cubic millimetre. In other cases a standard abbreviation is used, and occasionally a numeric code. To allow the staff greater flexibility, many of these sections also allow the data to be entered in full.

The menu driven structure enables staff quickly and conveniently to move between programs using a single key tap and therefore removes the need to learn complex computer syntax. Flexibility is important with any microbiology system and to assist in this up to 250 characters of free text are available for each specimen at all relevent stages of processing. This may be used for VDU and report form comments. Handwritten additions to reports are rare, reflecting the precision of the programming.

The hardware has proved to be generally reliable and flexible, and while the system was designed to allow easy return to manual methods if necessary, we have not experienced a major breakdown. Such hardware failures that have occurred have been a minor inconvenience and rectified within a few hours at most. The modular design of the system has meant that hardware failures in one section have not affected the rest of the system. Each unit of hardware may also be interchanged between benches if necessary.

Results inquiries, particularly concerning work under process, may take a considerable amount of time to service. At any one time the information available on a specimen may be a mixture of complete, preliminary, and incomplete, all of which may be useful for the immediate management of the patient. ${ }^{17} 18$ Even with the conventional data base systems this means referring to the individual benches within the laboratory. We have found this problem to have eased considerably for two reasons. Firstly, provisional reports may be generated very easily at any time of the day (consequently, we issue preliminary reports for over $80 \%$ of important isolates the day after specimen receipt). Secondly, the alphabetical daysheets contain a comprehensive record of all reports, including the current state of investigations of incomplete work. A specimen will therefore be listed on the daysheet each day with appropriate updating of investigations until finally reported. Such daysheets also allow great savings in storage space within the laboratory and obviate the need for bulky filing cabinets. For instance, the daysheets for one year's urine bacteriology (43000 specimens) may be held in one printout folder. A patient inquiry facility is also directly available for specimens under process at each of the bench microcomputers. The modular design does not at present allow a simultaneous inquiry of work under process to be made of different processing areas.

One of the more tangible benefits of the system to 
the clinical staff is the production of clear and concise report forms, which may be produced at any time of the day. Provisional reports, copies of reports for general practitioners and so on, and private patient copies for administration may be generated easily. We do not, however, have the facility for cumulative reporting.

The highly personalised nature of the software, while contributing to the acceptance and success of the system, would make transfer to other hospitals a difficult task. Over $80 \%$ of all specimens received are currently processed using the system described. Future plans include the early computerisation of the remaining processing areas and networking with the database, the storage of all results, and on line data capture from Bactec and gas-liquid chromatography equipment. The remaining processing areas will require only minor modifications to existing programs for their implementation.

In our experience microcomputers have proved to be a flexible, reliable, and inexpensive method of increasing the efficiency of a busy routine microbiology laboratory in the processing and reporting of investigations, thereby providing a better service to the clinical staff and patients.

We wish to thank Dr CD Plows, Mr BS Santy, and all the staff of the Department of Microbiology for their assistance and encouragement.

\section{References}

' Landowne RA. Data handling and reporting for microbiology specimens with a small laboratory computer system. J Clin Pathol 1981;34:637-41.

2 Farrar JL, Brogan TD, Moulding T, Taylor R, Page M. Use of a computer for producing microbiological reports and for data storage and processing. J Clin Pathol 1975;28:580-6.

' Ridgway GL, Batchelor J, Luton A, Barnicoat M. Data processing in microbiology: an integrated, simplified system. J Clin
Pathol 1980;33:744-9.

4 Perry J, Mitchison DA, Darrell JH. Use of the Phoenix system for bacteriology. J Clin Pathol 1983;36:104-9.

${ }^{5}$ Kobernick SD, Hendry P, Greer J. Coordinated computer reporting of microbiology data in the clinical laboratory. Am J Clin Pathol 1979;72:444-51.

- Ayliffe PF, Chalke R. A computer system to perform record handling and reporting for a hospital'microbiology laboratory. Med Lab Sci 1983;40:355-8.

7 Whitby JL, Blair JN. Data processing in hospital bacteriology: experience of 18 months trial. J Clin Pathol 1972;25:338-43.

${ }^{8}$ Goodwin CS, Smith BC. Computer printing and filing of microbiology reports. J Clin Pathol 1976;29:543-52.

` Alexander MK, Connigale J, Johnson T, Poulter IR, Wakefield J. A data processing system for hospital bacteriology. J Clin Pathol 1970;23:77-80.

10 Blair JN, Brown PP. An on-line computer system for hospital bacteriology: description of its development and comments after five years use. J Clin Pathol 1981;34:1132-7.

" Jorgenson JH, Holmes P, Williams WL, Harris JL. Computerisation of a hospital clinical microbiology laboratory. Am J Clin Pathol 1978;69:605-14.

12 Courcol RJ, Roussel-Delvallez M, Martin GR. A microcomputer system for clinical bacteriology: experience of 12 months trial. $J$ Clin Pathol 1982;35:881-7.

${ }^{13}$ Norbut AM, Foulis PR, Kreig AF. Microcomputer reporting and information system for microbiology. Am J Clin Pathol 1981;76:50-6.

14 Magee J. Microcomputers in microbiology. Med Lab Sci 1983;40:355-8.

is Clarkson DMcG, Thomas KL, Wynne-Williams. A microcomputer system for the collection and analysis of antibiotic sensitivity test data. J Clin Pathol 1982;35:444-51.

${ }^{16}$ McDonald T, Pearson NJ, Johnson F, O' Grady FW. Automated reading of the multipoint inoculated antibiotic sensitivity test. J Med Eng Technol 1981;5:243-5.

17 Ryan KJ. Computer systems in clinical microbiology. Clin Lab Med 1983;3:101-10.

${ }^{18}$ Drury J. Computers in the laboratory. $\mathrm{Br}$ Med $J$ 1983;287: 818-9.

Requests for reprints to: Mr B Downing, Chief Medical Laboratory Scientific Officer, Department of Microbiology, Good Hope General Hospital, Rectory Road, Sutton Coldfield, West Midlands B75 7RR, England. 